\title{
Decoherence and the rate of entropy production in chaotic quantum systems
}

\author{
Diana Monteoliva and Juan Pablo Paz \\ Departamento de Física "J.J. Giambiagi", FCEN, UBA, Pabellón 1, Ciudad Universitaria, 1428 Buenos Aires, Argentina
}

(November 13, 2018)

\begin{abstract}
We show that for an open quantum system which is classically chaotic (a quartic double well with harmonic driving coupled to a sea of harmonic oscillators) the rate of entropy production has, as a function of time, two relevant regimes: For short times it is proportional to the diffusion coefficient (fixed by the system-environment coupling strength). For longer times (but before equilibration) there is a regime where the entropy production rate is fixed by the Lyapunov exponent. The nature of the transition time between both regimes is investigated.
\end{abstract}

PACS numbers: 02.70.Rw, 03.65.Bz, 89.80.+h

Environment induced decoherence has been identified in recent years as one of the main ingredients in the transition from quantum to classical behavior. Classicality emerges as a consequence of the coupling of quantum systems to an environment which, in effect, dynamically enforces super-selection rules by precluding the stable existence of the majority of states in the Hilbert space of the system. The physics of decoherence has been thoroughly studied during the last few years both from the theoretical [1] and also from the experimental point of view [2]. As part of these studies it has been recognized that the decoherence process has rather unique properties for systems whose classical analogues are chaotic [3]. In fact, for classically chaotic systems decoherence has two very important features: First, decoherence restores the validity of the correspondence principle that for isolated chaotic systems could be violated in an absurdly short time-scale (see the Hyperion paradox in [4]). Thus, environment induced decoherence ensures that classical and quantum expectation values agree for a long range of times pushing towards infinity the "breakdown' time which would otherwise be present and would depend logarithmically on $\hbar$. This important feature was first conjectured in [3] and later studied in more detail in [4,5,6].

The second salient feature of decoherence for classically chaotic systems concerns the entropy production rate. In fact, in [3] it was also conjectured that, when decoherence is effective, there is a robust range of parameters for which the entropy production rate becomes independent of the strength of the coupling to the environment. In such regime, the rate is equal to the sum of positive Lyapunov exponents (the conjecture was modified in [7] where it was noticed that, in general, one should use appropriately averaged Lyapunov exponents).

In this letter we present the first conclusive numerical evidence supporting the above conjecture for open

*email addresses: monteoli@df.uba.ar, paz@df.uba.ar quantum systems evolving continuously in time (in the last few years the status of the conjecture was analyzed in part for some open quantum maps [8,9]7]). Our results show that Lyapunov exponent determines the value of the entropy production rate after an initial transient. We also clarify other important issues: First we show that during the initial transient the entropy production rate is proportional to the system-environment coupling strength. Second we analyze the transition between the two regimes. Our numerical results are consistent with a linear dependence of the transition time $t_{c}$ on the entropy of the initial state and with a logarithmic dependence on the coupling strength. Again, remarkably enough, our results validate once more the grossly oversimplified but very intuitive picture that was presented in [3].

The system we analyze is a quantum particle moving in a quartic double well potential under the action of an harmonic driving force. The Hamiltonian is $H=p^{2} / 2+V(x, t)$ with the potential $V(x, t)=$ $-B x^{2}+C x^{4} / 2+E x \cos (\omega t)$. This driven system has been extensively studied [10] and it is well known that for a wide range of parameters it has chaotic (mixed) behavior. In our studies we chose several sets of parameters in such a way that the stroboscopic phase space portrait is like the one shown in Figure 1 where one clearly sees the coexistence of islands of stability and a chaotic sea. The coupling of this system to an environment is modeled via the simplest (ohmic, high temperature) quantum Brownian motion model. In this case, the evolution of the reduced density matrix of the system follows a local master equation [14], which gives rise to the following equation for the Wigner function of the system:

$$
\begin{aligned}
\dot{W}=\{H, W\}_{P B} & +\sum_{n \leq 1} \frac{(-1)^{n} \hbar^{2 n}}{2^{2 n}(2 n+1) !} \partial_{x}^{(2 n+1)} V \partial_{p}^{(2 n+1)} W \\
& +2 \gamma \partial_{p}(p W)+D \partial_{p p}^{2} W
\end{aligned}
$$

The physical effects included in this equation are well understood. The first term in the right hand side is the Poisson bracket that induce classical evolution (Liouville flow). The second term carries the quantum corrections. 
The last two terms bring the influence of the environment generating, respectively, friction and diffusion. The regime of interest for us is the one where the friction term can be neglected $(\gamma \rightarrow 0$, and energy is approximately conserved) while the diffusive term, that produces decoherence is non-negligible ( $D=2 m \gamma k_{B} T=$ constant $)$.

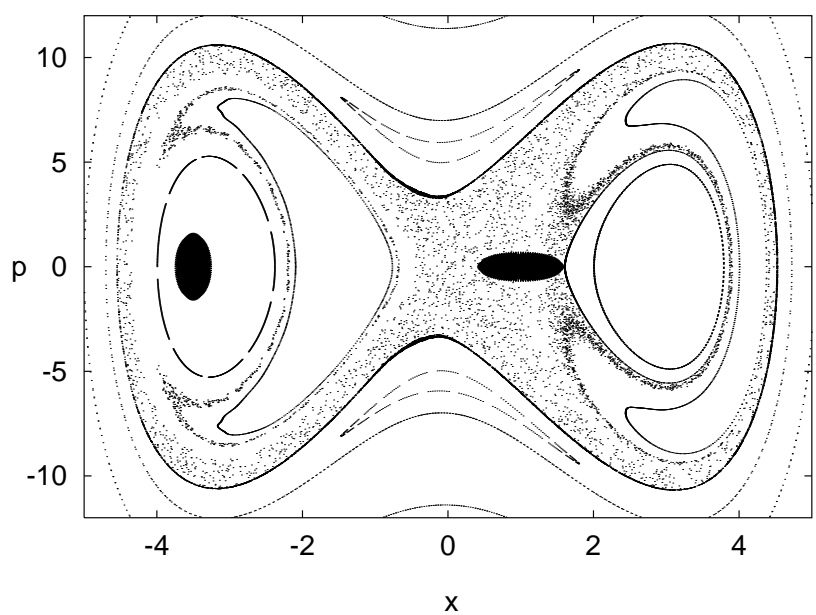

FIG. 1. Stroboscopic phase space portrait of the driven double well with parameters $B=10, C=0.5$, $E=1, \omega=5.35$. The black ellipses (one in a regular island and the other in the chaotic sea) show two of the initial states we considered (these are pure states and the boundary of the ellipses, whose area is $\pi \ln (20) \hbar \approx 9.4 \hbar$, corresponds to points where the Wigner function decreases to $1 / 20$ of its peak value). Throughout our studies we used $\hbar=0.1$.

Let us first qualitatively describe the most important effects we expect to test in our numerical study. We consider initial states with a smooth Gaussian Wigner function centered either in the chaotic sea or in a regular island as shown in Figure 1 (these two cases lead to drastically different results). Let us suppose first that the diffusion term in (1) is absent (i.e., the state evolves according to Schrödinger equation). For a smooth initial state the dominant term in (1) is the Poisson bracket. As Wigner function initially evolves following nonlinear classical trajectories it looses its Gaussian shape and develops tendrils while folding (this happens exponentially fast if the initial state is in the chaotic sea). As a consequence, gradients of $W$ increase and quantum corrections in (1) become important, inducing oscillations in the Wigner function. The decoherence producing term in (1) can be qualitatively understood as being responsible of two interrelated effects: On the one hand, the diffusion term tends to smooth out the Wigner function naturally reducing gradients. This washes out the oscillations in the Wigner function suppressing quantum interference. The time-scale characterizing the dissapearence of the fringes can be estimated using previous results [13]: Fringes with a characteristic wave-vector (along the $p$ axis of phase space) $k_{p}$ decay exponentially with a rate given by $\Gamma_{D}=D k_{p}^{2}$. Noting that a wave-packet spread over a distance $\Delta_{x}$ with two coherently interfering pieces generate fringes with $k_{p}=\Delta_{x} / \hbar$ one concludes that the decoherence rate is $\Gamma_{D}=D \Delta_{x}^{2} / \hbar^{2}$. This rate, depends linearly on the diffusion constant, and is one of the factors that contribute to the rate of entropy increase during the first phase of the evolution.

There is a second related aspect of decoherence which is drastically different for regular and chaotic systems. Thus, appart from suppressing the fringes, the diffusion term also tends to spread the regions where the Wigner function is possitive, contributing in this way to the entropy growth. But, as discussed in [3,7], the rate of entropy production distinguishes regular and chaotic cases. For regular states, decoherence should produce entropy at a rate which depends on the diffusion constant $D$. However, for chaotic states the rate should become independent of $D$ and should be fixed by the Lyapunov exponent. The origin of this $D$-independent phase can be understood using a simple minded argument (presented first in [3] and later discussed in a more elaborate way in [7]): Chaotic dynamics tends to contract the Wigner function along some directions in phase space competing against diffusion. These two effects may balance each other giving rise to a critical width below which Wigner function cannot contract. This local width should be approximately $\sigma_{c}^{2}=2 D / \lambda$ (being $\lambda$ the local Lyapunov exponent). Once this critical size has been reached, the contraction stops along the stable direction while the expansion continues along the unstable one. Therefore, the area covered by the Wigner function grows exponentially in time and, as a consequence, entropy grows linearly with a rate fixed by the Lyapunov exponents.

Here, we will present solid numerical evidence supporting the existence of this $D$-independent phase. For simplicity, instead of looking at the von Neuman entropy $\mathcal{H}_{V N}=-\operatorname{Tr}\left(\rho_{r} \log \rho_{r}\right)$ we examine the linear entropy, defined as $\mathcal{H}=-\log \left(\operatorname{Tr}\left(\rho_{r}^{2}\right)\right)$, which is a good measure of the degree of mixing of the system and sets a lower bound on $\mathcal{H}_{V N}$. The above argument concerning the role of the critical width $\sigma_{c}$ may appear as too simple but captures the essential aspects of the dynamical process. Indeed, the master equation can be used to show that

$$
\dot{\mathcal{H}}=2 D\left\langle\left(\partial_{p} W\right)^{2}\right\rangle /\left\langle W^{2}\right\rangle
$$

where the bracket denotes an integral over phase space. The right hand side of this equation is proportional to the mean square wave-number computed with the Fourier transform of the Wigner function. This implies that the entropy production rate is closely related to the phase space structure present in the Wigner distribution. Thus, the $D$-independent phase begins at the time when the mean square wave-length along the momentum axis scales with diffusion as $\sqrt{D}$ (as $\sigma_{c}$ does). This behav- 
ior cancels the diffusion dependence of $\dot{\mathcal{H}}$ which becomes entirely determined by the dynamics.

Appart from analyzing the $D$-independent phase of entropy production we will analyze the nature of the the transition time between the diffusion dominated and the chaotic regime. This time $t_{c}$ can also be naively estimated along the lines of the previous argument: The time for which the spread of the Wigner function approaches the critical one is $t_{c} \approx \lambda^{-1} \log \left(\sigma_{p}(0) / \sigma_{c}\right)$. Thus, one expects $t_{c}$ to depend logarithmically on the diffusion constant and on the initial spread of the Wigner function (for Gaussian initial states the spread depends exponentially on the initial entropy, therefore $t_{c}$ should vary as a linear function of the initial entropy). Our numerical work is devoted at testing these intuitive ideas.

We solved numerically the master equation using two different methods (that give similar results). First, we computed the Wigner function numerically solving equation (11) using a third order pseudo-spectral method [12]. For this, we used a phase space grid of variable size and adaptive time-steps (a similar method was used in [5]). Second we obtained approximate solutions to (1) using a different strategy: We computed the Floquet states of the original (isolated) driven system and obtained the master equation in the Floquet basis 11]. This equation has a rather simple form when averaged over one driving period. In such case, it can be numerically solved by usual means (the limiting factor is the number of Floquet states that are required). In what follows we present our results for the entropy behavior (plots are obtained using the first of the two numerical methods, more details and other results will be presented elsewhere [15]).

The drastic difference between the behavior of the entropy production rate for regular and chaotic initial conditions is clearly displayed in Figure 2. Regular initial conditions produce entropy at a rate linearly dependent on the diffusion coefficient. This is precisely what we expect when the entropy production is due to: (i) the destruction of interference fringes, (ii) the slow increase in the area covered by the possitive Wigner function. The oscillations evident in Figure 2 have some distinguishing features: they have the frequency of the driving force and both their amplitude and their phase are $D$-independent (this is true for the regular initial state and for the initial transient of the chaotic state). These oscillations can be shown to be related both to the change in orientation of the fringes (decoherence is more effective if fringes are aligned along the $p$ axis) and to the change in spread of the Wigner function in the momentum direction induced by the dynamics. When the initial state is centered in the chaotic sea, the initial transient is followed by a regime where the rate $\dot{\mathcal{H}}$ becomes independent of the value of the diffusion constant (if $D$ is not too small, see below). Moreover, the numerical value of the rate oscillates around the average local Lyapunov exponent computed by averaging over an ensemble of classical tra- jectories weighted by the initial Wigner function (In [7] the use of a more elaborate averaging scheme was suggested, we will compare these approaches elsewhere [15]).
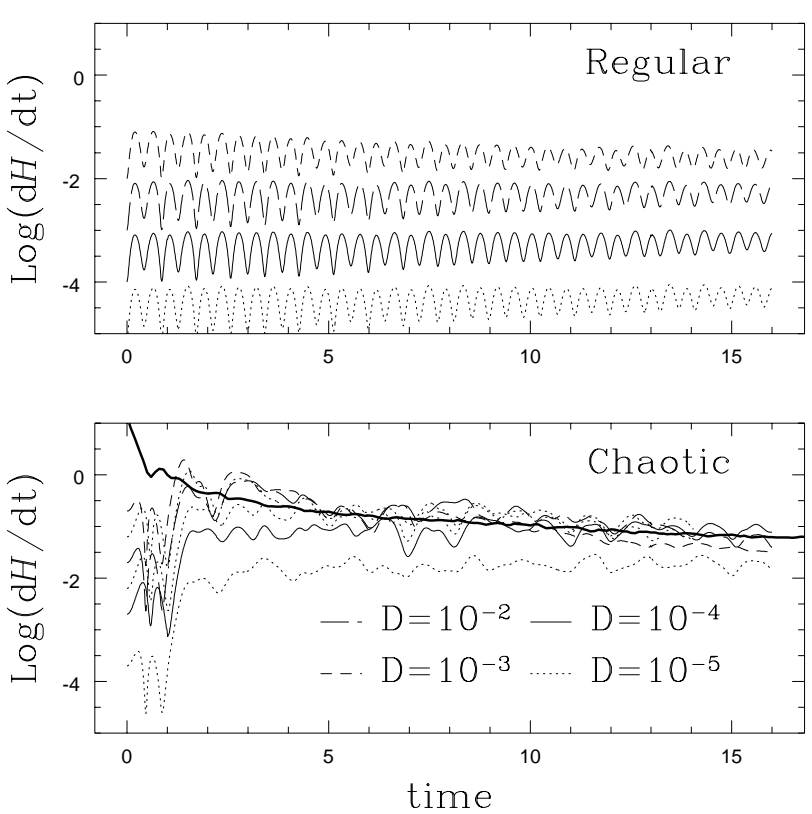

FIG. 2. Entropy production rate vs time (in units of the driving period). The bold curve is the (time dependent) Lyapunov exponent. The linear dependence of the rate on $D$ appears in the graph at the top (regular initial state) and during the initial transient in the lower plot. In this case (initial state in the chaotic sea) the rate becomes independent on diffusion and is equal to the Lyapunov exponent (if $D$ the is not too small, see text).

It is remarkable that for long times the entropy production rate is indeed fixed just by the dynamics becoming independent of $D$ (after all, the entropy production is itself a consequence of the coupling to the environment but the value of the rate becomes independent of it!). The result presented in Figure 2 was shown to be robust under changes of initial conditions and other parameters characterizing the classical dynamics. There are two limitations for the above results to be obtained. On the one hand the diffusion constant cannot be too strong: In that case the system heats up too fast and entropy saturates, making the numerical simulation unreliable. On the other hand, diffusion cannot be too small either: If that is the case decoherence could become too weak and the interference fringes could persist over many oscillations (the minimal value of $D$ required for efficient decoherence could be estimated as follows: if the Wigner function is coherently spread over a region of size $\Delta_{x} \approx 10$, we would need a diffusion constant larger than $D_{\text {min }} \approx \hbar^{2} / \Delta_{x}^{2} \approx 10^{-4}$ for the environment to be able to wash out the smallest fringes in one driving period. In fact, Figure 2 shows that when $D=10^{-5}$ the entropy production rate is one order of magnitude smaller 
than the one corresponding to $D=10^{-4}$.
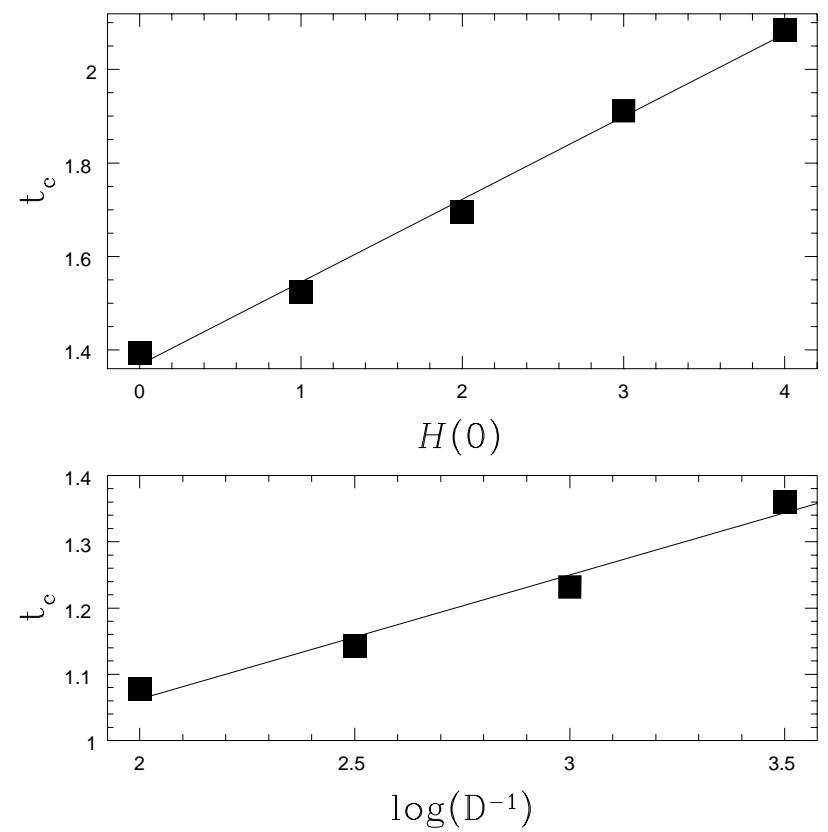

FIG. 3. The transition time between the diffusion dominated regime and the one where the entropy production rate is set by the Lyapunov exponent is shown to depend linearly on entropy (top) and logarithmically on the diffusion constant (bottom). Numerical results were obtained using the parameters: $B=10, C=0.5, E=10, \omega=6.16, D=10^{-3}$ (top), $\mathcal{H}(0)=0$ (bottom)

It is interesting to remark that while the simple picture presented in [3] is in good qualitative agreement with our results, the arguments presented in that paper are too simple to include some important effects we found. In particular, the oscillatory nature of the rate was completely overlooked in [3]. However, having said this, it is still possible to test some simple results obtained in [3] for the transition time between the diffusion dominated regime and the one where the rate is fixed by the Lyapunov exponent. For this purpose we performed two related studies. First we analyzed the dependence of the transition time $t_{c}$ on the diffusion constant. Of course, there is some ambiguity in the definition of the transition time because of the oscillatory nature of the rate. Here, we defined $t_{c}$ as the time for which the rate reaches some value after the initial transient (fortunately the value of the rate before and after the transient differ by two orders of magnitude making this a reasonable definition). Second, we analyzed the behavior of the rate as a function of the entropy of the initial state (i.e., on the spread of the initial Wigner function). Again, we used the same definition of $t_{c}$ (for this study we considered a different set of parameters than the one used to construct Figure 1 to assure that the chaotic sea can accommodate initial states with linear entropy up to $\mathcal{H}=4$ ). With this results we obtained the plots in Figure 3 where the lin- ear dependence of $t_{c}$ on the initial entropy (mixed initial states produce entropy at a smaller rate) and its logarithmic dependence on the diffusion constant are shown.

Entropy production, a consequence of the entangling interactions between the system and its environment, plays a central role in studies of decoherence (for example, it is esential to find the "pointer states" of the system using the "predictability sieve" [16]). Here, we presented results supporting the point of view stating [4] that entropy production rate during decoherence could also be used as a diagnostic for quantum chaos. On the other hand these results also make evident the fact that, for quantum systems that are classically chaotic, the nature of the classical limit induced by decoherence is rather peculiar (and quite different from the one corresponding to regular systems). Indeed, this limit exhibits an unavoidable source of unpredictability, being the rate at which information is lost into the environment entirely fixed by the chaotic nature of the Hamiltonian of the system.

This work was supported by grants from Anpcyt (PICT 01014), Ubacyt and Conicet.

[1] see for example W. H. Zurek, Physics Today 44, (1991) N 10, 36; Giulini et al Decoherence and the appearance of the classical world in quantum theory (Springer Verlag, Berlin, 1996).

[2] J. M. Brune et al, Phys. Rev. Lett. 77, 4887 (1996), see also Phys Today51, 7, 36 (1998).

[3] W. H. Zurek and J. P. Paz, Phys. Rev. Lett. 72 (1994) 2508.

[4] W. H. Zurek and J. P. Paz, Physica 83D (1995) 300.

[5] S. Habib, K. Shizume and W. H. Zurek, Phys. Rev. Lett. 80 (1998) 4361.

[6] J. Gong and P. Brumer, Phys. Rev. E60 (1999) 1643.

[7] A. K. Pattanayak, Phys. Rev. Lett. 83 (1999) 4526.

[8] K. Shiokawa and B. L. Hu, Phys. Rev E52 (1995) 2497.

[9] P. A. Miller and S. Sarkar, Phys. Rev. E58 (1998) 4217; 60 (1999) 1542.

[10] W. A. Lin and L. E. Ballentine, Phys. Rev. Lett. 65 (1990) 2927; Phys. Rev.A45 (1992) 3637.

[11] T. Dittrich, B. Oelschlägel and P. Hänggi, Europh. Lett. 22 (1993) 5.

[12] M. Feit, J. Fleck Jr. and A. Steiger, J. Comp. Phys. 47 (1992) 412.

[13] J. P. Paz, S. Habib and W. H. Zurek, Phys. Rev. D47 (1992) 488.

[14] A. O. Caldeira and A. J. Leggett, Physica 121A (1983) 587; B. L. Hu, J. P. Paz and Y. Zhang Phys. Rev. D45 (1992) 2843.

[15] D. Monteoliva and J. P. Paz, in preparation

[16] W. H. Zurek, S. Habib and J. P. Paz, Phys. Rev. Lett. 70 (1993 1187; J. P. Paz and W. H. Zurek, Phys. Rev. Lett. 82 (1999) 5181. 